\title{
Improvement of the Modified Dry-Cup Method for Determination of the Diffusion Coefficients of Gaseous Pollutants in Building Materials
}

\author{
José J. Gonçalves ${ }^{a}$, Gabriela V. Silva ${ }^{b}$, \\ Armando S. Santos ${ }^{c}$ and Eduardo O. Fernandes ${ }^{d}$ \\ IDMEC, Institute of Mechanical Engineering, University of Porto, \\ Rua Dr. Roberto Frias, 4200-465 Porto, \\ Portugal \\ ajpg@fe.up.pt, ${ }^{b} g v s @ f e . u p . p t,{ }^{c} a s a n t o s @ f e . u p . p t,{ }^{d}$ eof@fe.up.pt
}

\author{
Keywords: Building Materials, Diffusion Coefficient, Indoor Air Quality, Mass Diffusion, Volatile \\ Organic Compounds
}

\begin{abstract}
Diffusion and partition coefficients are key parameters for the characterization of building materials as sources and sinks of air pollutants indoors. A simple and rapid experimental method for direct measurement of the diffusion coefficient $(D)$ was presented in a previous article [1]: the modified dry cup method. This method was based upon two existent methodologies, the passive sampling on Tenax TA and the dry cup method, proposed by Haghighat et al. [2]. In the present article, some improvements were made in the experimental set-up in order to obtain more precise results. The previous cup was modified by increasing the number of Tenax tubes, connected to the bottom of the cup, from one to five. The results obtained with both cups were compared. Measurements with single compounds and with three compounds simultaneously were compared in order to evaluate the influence of competition between molecules of different compounds.
\end{abstract}

\section{Introduction}

As a result of the time people spend indoors, estimated at around $90 \%$, and poor levels of ventilation in the spaces, imposed in large part by energy issues, the problem of poor indoor environmental quality has increased. Building materials, among other sources, contribute to these problems due to emissions of volatile organic compounds (VOCs).

The measurement and prediction of the emission rates of VOCs from building materials have been the subject of intense research. In this context, the development of mathematical models for prevision of the concentration of pollutants indoors requires a previous study of the behaviour of the materials $v s$ pollutants in order to quantify the involved parameters, represented by the diffusion and partition coefficients [3]. The available data in the literature for those coefficients is still scarce and, in general, have highly dispersed values; depending upon the method used for their determination.

The main objective of the present work was to improve the modified dry cup method for the determination of $D$ [1]. The previous cup was modified by increasing the number of Tenax tubes, connected to the bottom of the cup, from one to five. Measurements made with single compounds, and with a mixture of three compounds simultaneously, were compared in order to evaluate the influence of competition between molecules of different compounds.

\section{Research Methods}

Diffusion is the mode of mass transfer of a compound caused by a concentration gradient. Diffusive transport of gases in building materials involves a complex variety of processes including molecular, Knudsen and surface diffusion within the porous interstices [4]. Several experimental techniques have been used to estimate the diffusion coefficients, being the three major methods for 
the measurement of diffusion coefficients of building materials: the wet cup [5], the twin chamber [6] and the porosity test method [4].

The simplest approach used to measure the diffusion coefficient of building materials is the wet cup method [5, 7]. It is based upon Fick's $1^{\text {st }}$ law [8]. The main disadvantage of this method is that the liquid VOC placed in the cup creates a concentration of saturation, which is unrealistically high for indoor air quality application, with values of around dozens of $\mathrm{g} / \mathrm{m}^{3}$ [2]. Thus, it is expected that the cup method will overestimate the diffusion coefficient. Haghighat et al. [2] proposed the use of this method with a modification, and called it the "dry cup method", in contrast to the old "wet cup method". In the dry cup method, a desiccant, e.g. activated carbon, is placed within the cup in order to create zero vapour pressure. The test is performed by sealing the test specimen to the open mouth of a cup containing the desiccant, and placing the assembly into a controlled atmosphere (Fig. 1). The test unit is weighed periodically, and the weight is plotted as a function of time.

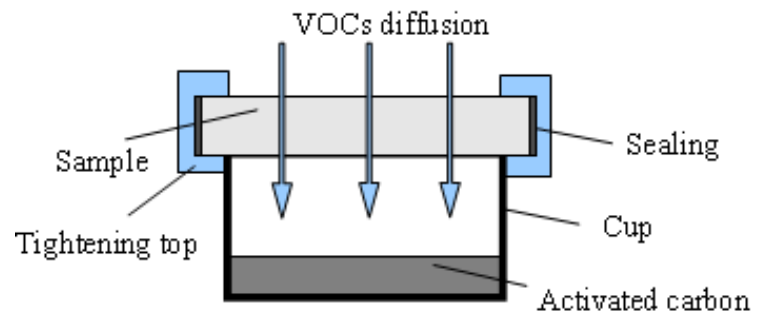

Figure 1

Scheme of the dry cup used for diffusion tests

Experiments were performed using this method, but the results showed that the time needed to achieve data which was representative of the permanent regime was too long. In addition, experiments have proved to be extremely sensitive to the presence of water vapour; up to the point of being a dominant element in the global transport. Separating the effects of relative humidity in the dry cup method is very difficult, particularly when the adsorbent has a great affinity for water vapour. In response to this difficulty, the method of determining the mass transfer of VOCs, then gravimetric, was replaced by an analytical method, by means of passive sampling, in which the mass transferred is collected by adsorption on Tenax TA tubes and subsequently quantified by gas chromatography - mass spectrometry (GC-MS).

\section{Modified dry cup method}

This method can be considered a derivative of the "dry cup", as suggested by the schematic representation of Fig. 2. In this method, the Tenax TA tube is connected to the bottom of the cup (modified) and acts as a desiccant to adsorb the compounds that diffuse through the material, and is then analyzed by GC-MS. This method allows the study of several compounds simultaneously.

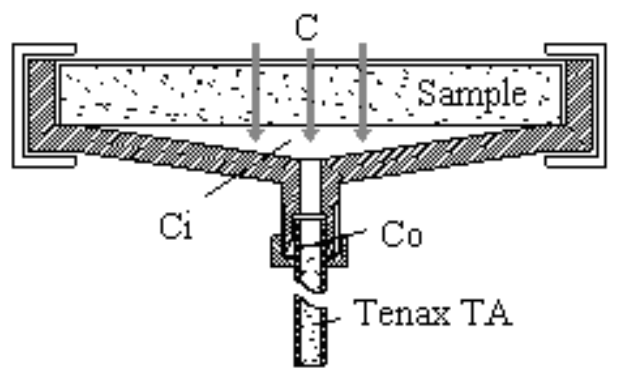

Figure 2

Scheme of the modified dry cup method

used for diffusion tests 
Passive samplers are based upon the mass transport associated with a concentration gradient that, in dynamic equilibrium, can be expressed by Fick's first law:

$$
\dot{m}=-D A \frac{d C}{d x}
$$

Assuming that the adsorbent operates with a high capacity for adsorption, a performance in the permanent regime is expected. In this condition, flow of VOC can be determined, knowing the mass of the adsorbed compound, $\delta m$, during a certain time of sampling, $\delta t(\dot{m}=\delta m / \delta t)$.

Assuming negligible resistance to diffusion in the intermediate air layer, Eq. 1 for the system represented in Fig. 2 can be expressed as:

$$
\dot{m}=\frac{D_{\text {air }} A_{t}\left(C_{i}-C_{0}\right)}{d} .
$$

where $D_{\text {air }}$ is the diffusion coefficient of the compound in air, $A_{t}$ is the cross-sectional area of sampling tube (Tenax tube), $C_{i}$ is the concentration in the intermediate region (between the material and the sampling tube), $C_{0}$ is the concentration of the compound on the surface of the adsorbent and $d$ is the length of the layer of air from the sorbent bed to the end of the sampling tube. Ideally, $C_{0}=0$ ("zero sink" conditions). Considering that the volume of the intermediate region is very small $\left(0.0000141 \mathrm{~m}^{3}\right)$, and thus the mass that remains in the intermediate region is only about $1 \%$ of the mass adsorbed by the Tenax tubes, it was assumed that the adsorbed mass in the Tenax tubes was the total mass transferred. The concentration $C_{i}$ results from diffusion of mass through the porous material between the intermediate region and the environment inside the chamber at concentration, $C$. This can be expressed by the equation:

$$
\dot{m}=D_{e} A \frac{\left(C-C_{i}\right)}{L}
$$

where $D_{e}$ is the diffusion effective coefficient of material with area, $A$, and thickness, $L$. Assuming a permanent regime, the effective diffusion coefficient results in:

$$
D_{e}=D_{a r} \cdot \frac{L}{d} \cdot \frac{A_{t}}{A} \cdot \frac{\left(C_{i}-C_{0}\right)}{\left(C-C_{i}\right)}
$$

The concentration $\left(\mathrm{C}_{\mathrm{i}}\right)$ is the result of mass diffusion between the interior of the test chamber and the inside of the cup through the porous material that makes up the boundary between these two spaces. The concentration $C_{i}$ is obtained from Eq. 2, and $D_{e}$ from Eq. 4.

\section{Experimental Set-Up}

The experimental set-up has been presented previously [1]. Later, the cup was modified and now accommodates 5 Tenax tubes, in order to obtain more precise results. The cup is placed in a test chamber made of stainless steel and with a capacity of $0.255 \mathrm{~m}^{3}$. It is provided with means for the control of temperature and relative humidity. All experiments are performed within $(23 \pm 1){ }^{\circ} \mathrm{C}$ and $(45 \pm 5) \%$. No pressure gradients across the material samples could be detected, because the cells were constantly ventilated with air. The experimental chamber was cleaned before starting an experiment. 
A thermal desorption system (Dani, model STD 33.50) on line with a gas chromatograph (Agilent Technologies, model 6890N), coupled to a mass selective detector (Agilent Technologies, model 5973), for VOC identification and quantification was used.

For the diffusion experiments, three VOCs (m-Xylene, n-Octane and Cyclohexane) were chosen having differing properties. The properties of the compounds studied, namely the values of the diffusion coefficient in air [9], are presented in Table 1. Measurements of single compounds and of the three compounds simultaneously were compared in order to evaluate the influence of competition between molecules of different compounds.

Table 1

Properties of m-Xylene, n-Octane and Cyclohexane

\begin{tabular}{llll}
\hline Properties & Cyclohexane & n-Octane & m-Xylene \\
\hline Molecular formula & $\mathrm{C}_{6} \mathrm{H}_{12}$ & $\mathrm{CH}_{3}\left(\mathrm{CH}_{2}\right)_{6} \mathrm{CH}_{3}$ & $1,3-\left(\mathrm{CH}_{3}\right)_{2}\left(\mathrm{C}_{6} \mathrm{H}_{4}\right)$ \\
Molecular weight $\left[\mathrm{g} \cdot \mathrm{mol}^{-1}\right]$ & 84.16 & 114.2 & 106.17 \\
Vapour pressure at $23^{\circ} \mathrm{C}[\mathrm{Pa}]$ & 12800 & 1674. & 1003 \\
Boiling point $\left[{ }^{\circ} \mathrm{C}\right]$ & 80.7 & 125.6 & 139.1 \\
Diffusion coefficient in air $\left[\mathrm{m}^{2} \cdot \mathrm{h}^{-1}\right]$ & 0.0284 & 0.0227 & 0.0252 \\
\hline
\end{tabular}

The building materials used for testing were the same as those used in previous studies [1], in order to obtain comparable data (Table 2). The experiments were performed on unfinished cork parquet, gypsum board and polyvinyl chloride (PVC). All of the samples were placed in a chamber (at $23^{\circ} \mathrm{C}$ and $45 \%$ of $\mathrm{RH}$ ) for stabilization before testing.

Table 2

Properties of the tested building materials

\begin{tabular}{|c|c|c|c|}
\hline Material & $\begin{array}{l}\text { Thickness } \\
\text { [mm] }\end{array}$ & $\begin{array}{l}\text { Density } \\
{\left[\mathrm{kg} / \mathbf{m}^{3}\right]}\end{array}$ & Description of the materials \\
\hline PVC floor covering & 2,07 & 1577 & Solid PVC \\
\hline Cork parquet & 1.85 & 435 & $\begin{array}{l}\text { Cork flooring material without } \\
\text { finishing }\end{array}$ \\
\hline Gypsum board & 9.73 & 755 & Covered with cardboard paper \\
\hline
\end{tabular}

\section{Results and Discussion}

The diffusion coefficients obtained using the modified dry cup method, for one sampling point and five sampling points, are presented in Table 3.

The improvement in the cup from one point of sampling to five points of sampling, did not indicate a large difference between the value of the diffusion coefficient calculated with one or five sampling tubes; which eliminated the possibility of reaching saturation with a single tube. The calculation for five tubes is more reliable because it is based upon average values and permits the identification of outliers (performed by application of statistical Dixon test at a significance level of $0.05)$.

The values in Table 4 present those of diffusion coefficients obtained using the modified dry cup method for five sampling points, with exposure to single compounds and to the three compounds simultaneously. The standard deviation of the multiple analysis after identification of outliers is presented. 
Table 3

Experimental values of D obtained by single compound for modified dry cup method, for one and five Tenax tubes

\begin{tabular}{lcccccc}
\hline & \multicolumn{6}{c}{$\mathbf{D}\left[\times \mathbf{1 0}^{-\mathbf{6}} \mathbf{~}^{\mathbf{2}} / \mathbf{h}\right]$} \\
\cline { 2 - 7 } & \multicolumn{2}{c}{ Xylene } & \multicolumn{2}{c}{ Octane } & \multicolumn{2}{c}{ Cyclohexane } \\
\hline PVC floor covering & 0.17 & 0.58 & 0.14 & 0.12 & 0.17 & 0.10 \\
Cork parquet & 5.3 & 6.6 & 4.0 & 4.6 & 7.1 & 10.1 \\
Gypsum board & 27.2 & 27.9 & 26.2 & 28.4 & 39.1 & 40.8 \\
\hline
\end{tabular}

Table 4

Experimental values of $D$ obtained by exposure to single and multiple compounds for modified dry cup method, for five Tenax tubes

\begin{tabular}{lcccccc}
\hline & \multicolumn{9}{c}{$\mathbf{D}\left[\times \mathbf{1 0}^{-\mathbf{6}} \mathbf{~ m}^{\mathbf{2}} / \mathbf{h}\right]$} \\
\cline { 2 - 7 } & Single & Multiple & Single & Multiple & Single & Multiple \\
PVC floor covering & $0.58 \pm 0.06$ & $0.27 \pm 0.02$ & $0.12 \pm 0.01$ & $0.07 \pm 0.01$ & $0.10 \pm 0.01$ & $0.18 \pm 0.02$ \\
Cork parquet & $6.6 \pm 0.4$ & $5.5 \pm 0.3$ & $4.6 \pm 0.7$ & $4.4 \pm 0.3$ & $10.1 \pm 1.9$ & $7.6 \pm 0.2$ \\
Gypsum board & $27.9 \pm 0.7$ & $26.9 \pm 1.0$ & $28.4 \pm 2.9$ & $26.2 \pm 1.3$ & $40.8 \pm 2.1$ & $32.7 \pm 3.0$ \\
\hline
\end{tabular}

The results of experiments on the determination of $\mathrm{D}$ upon exposing the material to a single compound, or to the three compounds simultaneously, show some differences. The $t$-student test at a significance level of 0.05 was applied to the values obtained for single and multiple exposure in order to evaluate the differences. For PVC, all of the values of D obtained are statistically different and without a constant pattern: for the compound, xylene, a high value was obtained in a single exposure but, for cyclohexane, a high value of $\mathrm{D}$ was obtained in multiple exposures. For the cork sample and for gypsum board, the results of D are higher for single exposure tests. However, only the values obtained for the pairs, cork/xylene, cork/cyclohexane and gypsum board/cyclohexane, are statistically different.

For a more complete analysis, the error associated with a set of different tests should be determined and only then can the influence of the effects of single/multiple exposure be assessed. However, even when statistically different, the values obtained are of the same order of magnitude. Moreover, the results obtained experimentally by using the modified dry cup method fall within the existing literature values; obtained by using other methods, such as that of the twin chambers [10], subject to similar gradients of concentrations, and close to the values of indoor environments.

\section{Conclusions}

The results obtained by using the modified dry cup method show that this method is a sensitive method that allows the obtention of results within a short time. The attempt to obtain diffusion coefficients by exposing materials to several compounds simultaneously, has not yet provided reliable results. More experiments are needed before drawing conclusions, but the results obtained are promising, as the diffusion coefficient obtained for the single and multiple exposure are of the same order of magnitude. 


\section{Acknowledgement}

The authors wish to thank the financial support of FCT for the project "Diffusion of organic vapours in building materials indoors. Influence of the material microstructure". (PTDC/ EMEMFE/71915/2006).

\section{References}

[1] J.J. Gonçalves, A.S. Santos, G.V. Silva and E.O. Fernandes, "Presentation of a new method for determination of diffusion coefficients of gaseous pollutants in cladding wall materials indoors”. Defect and Diffusion Forum, Vols. 297-301 (2010).

[2] F. Haghighat, C.S. Lee and W.S. Ghaly, Measurement of diffusion coefficients of VOCs for building materials: review and development of a calculation procedure. Indoor Air, 12, 81-91 (2002).

[3] Meininghaus R. Gunnarsen L. and Knudsen HN. 2000. "Diffusion and sorption of volatile organic compounds in building materials - Impact on indoor air quality," Environmental Science Technology. 34: 3101-3108.

[4] Blondeau, P., Tiffonnet, A. L., Damian, A., Amiri, O. and Molina, J. L., 2003. Assessment of contaminant diffusivities in building materials from porosimetry tests. Indoor Air, 13 (3), 302310.

[5] Kirchner, S., Badey, J.R., Knudsen, H.N., Meininghaus, R., Quenard, D., Sallee, H. and Saarinen, A., 1999. Sorption capacities and diffusion coefficients of indoor surface materials exposed to VOCs: proposal of new test procedures. In: Proceedings of the $8^{\text {th }}$ International Conference on Indoor Air Quality and Climate - Indoor Air 1999, Edinburgh, Vol. 1, pp. 430 -435 .

[6] Meininghaus R., Uhde E., 2002. Diffusion studies of VOC mixtures in a building material, Indoor Air, 12, 215-222.

[7] Guindeira, M., Santos, A. and Oliveira Fernandes, E., 2005. Experimental studies on diffusion coefficients of volatile organic compounds for building materials. In: Proceedings of the $10^{\text {th }}$ International Conference on Indoor Air Quality and Climate - Indoor Air 2005, Beijing, pp. 1974.

[8] Crank, J., 1975. The Mathematics of Diffusion. Oxford University Press.

[9] Seo, J., Kato, S., Ataka, Y., Zhu, Q., "Evaluation of Effective Diffusion Coefficient in Various Building Materials and Absorbents by Mercury Intrusion Porosimetry". (2005), Indoor Air 2005, The $10^{\text {th }}$ International Conference on Indoor Air Quality and Climate.

[10] Richard C., Neil C., Fardal J., Little J., Ying X. "Determination of Sorption Parameters for 36 VOC/Material Combinations". Final Report. United States Environmental Protection Agency. 2006. 